Посвящается 100-летию со дня рождения одного из основателей воронежской математической школь -

М. А. Красносельского

\title{
ОБ ИСТОРИИ ПОЛОЖИТЕЛЬНЫХ ОПЕРАТОРОВ (1900-е - 1960-е гг.) И ВКЛАДЕ М. А. КРАСНОСЕЛЬСКОГО ${ }^{1}$
}

\author{
Е. М. Богатов \\ (Статъя представлена членом редакционной коллегии Ю. П. Вирченко) \\ Старооскольский технологический институт им. А. А. Угарова (филиал) Национального \\ исследовательского технологического университета «МИСиС», \\ г. Старый Оскол, 309516, Россия \\ Филиал Национального исследовательского технологического университета «МИСиС» \\ в г. Губкине Белгородской области, \\ г. Губкин, 309180, Россия \\ E-mail: embogatov@inbox.ru
}

Аннотация. Целью работы является изучение вклада зарубежных и отечественных математиков, в особенности М. А. Красносельского, в развитие теории линейных и нелинейных положительных операторов за период с середины 1900-х гг. до конца 1960-х гг.

Метод. Исследование основано на анализе оригинальных работ О. Перрона, Ф. Г. Фробениуса, Р. Ентча, П. С. Урысона, М. Г. Крейна, М. А. Рутмана, М. А. Красносельского и др. в контексте общемирового процесса развития функционального анализа.

Результат. Вклад отечественных учёных в области положительных операторов оказался больше, чем вклад остальной части мирового математического сообщества в рассматриваемый период. Советские математики М. Г. Крейн и его ученик М. А. Рутман в 1940-е гг. создали теорию конусов и линейных положительных операторов $A$ в бесконечномерном пространстве и применили её к исследованию разрешимости уравнений вида $A x=\lambda x$. Благодаря усилиям другого ученика М. Г. Крейна - М. А. Красносельского - с середины 1950-х гг. теория положительных операторов приобрела своё значение, как общий метод для решения широкого класса задач качественного характера, относящихся к анализу нелинейных операторных уравнений (в том числе, доказательство новых теорем о неподвижной точке и структуре спектра положительного оператора $A$, исследование бифуркационных значений параметра $\mu$ в уравнении вида $x=A(x, \mu)$, обоснование метода последовательных приближений для уравнения $A x=\lambda x$ с нелинейным оператором $A$ в конусе банахова пространства и т.п.). Кроме того, в рамках развитой М. А. Красносельским теории удалось решить ряд задач прикладного характера.

Обсуждение. Анализ достижений в области положительных операторов показал, что в отдельно взятой стране (CCCP) могут быть сформированы условия для успешного создания и развития целого научного направления. Огромное значение здесь имеет масштаб учёных, стоящих у истоков этого направления - М. Г. Крейна и М. А. Красносельского.

Ключевые слова: история нелинейного функционального анализа, теорема Перрона - Фробениуса, теорема Ентча, конусная теорема Красносельского, положительные операторы, теория конусов, уравнение Гаммерштейна, уравнение Урысона.

Благодарности: Работа выполнена при финансовой поддержке РФФИ, проект 20-011-00402.

Для цитирования: Богатов Е. М. Об истории положительных операторов (1900-е - 1960-е гг.) и вкладе М. А. Красносельского. Прикладная математика \& Физика, 52(2): 105-127.

DOI 10.18413/2687-0959-2020-52-2-105-127.

\footnotetext{
${ }^{1}$ Работа представляет собой расширенный и дополненный вариант доклада на Воронежской зимней математической школе С. Г. Крейна - 2020 [Богатов, 2020], а также выступлений на XXV годичной научной конференции ИИЕТ PAH им. С. И. Вавилова, секция История математики, [Богатов, 2019 а] и на XVI международной конференции «Алгебра, теория чисел и дискретная геометрия: современные проблемы, приложения и проблемы истории [Богатов, 2019 b]»
} 


\title{
ON THE HISTORY OF THE POSITIVE OPERATORS (1900s - 1960s) AND THE CONTRIBUTION OF M. A. KRASNOSEL'SKII
}

\author{
Egor Bogatov
}

(Article submitted by a member of the editorial board Yu. P. Virchenko)

Stary Oskol Technological Institute of National Research University of Science and Technology «MISIS», Stary Oskol, 309516, Russia;

Branch of National Research University of Science and Technology «MISIS» in Gubkin town of Belgorod Region Gubkin, 309180, Russia

E-mail: embogatov@inbox.ru

Received May 20, 2020

Abstract. Goal. The aim of the work is studying of the contribution of foreign and domestic mathematicians, in particular M. A. Krasnoselskii, to the development of the theory of linear and nonlinear positive operators for the period from the mid-1900s until the end of the 1960s.

Method. The study is based on an analysis of original works of O. Perron, G. Frobenius, R. Jentzsch, P. S. Urysohn, M. G. Krein, M. A. Rutman, M. A. Krasnoselskii and others in the context of the global process of development of functional analysis.

Result. The contribution of domestic scientists in the field of positive operators was larger than that of the rest of the world mathematical community in the period under review. Soviet mathematicians M. G. Krein and his student M. A. Rutman in the 1940s created the theory of cones and linear positive operators $A$ in space of infinite dimension. They applied this theory to the study of the solvability of equations of the form $A x=\lambda x$. Thanks to the efforts of another Krein student - M. A. Krasnoselskii - the theory of positive operators has become a general method for solving a wide class of problems of a qualitative nature, related to the analysis of nonlinear operator equations, since the mid 1950s (proof of new fixed point theorems and theorems about the spectrum structure of the operator $A$ ), investigation of the bifurcation value of the parameter $\mu$ in the equation $x=A(x, \mu)$, substantiation of the successive approximations method for the equation $A x=\lambda x$ in a cone of Banach space for nonlinear operator $A$ and so on). Besdides, in the framework of the theory created by Krasnoselskii, a number of important applied problems were solved.

Discussion. Analysis of developments in the field of positive operators showed that in one country (USSR) may be formed conditions for the successful creation and development of a separate scientific field. Of great importance here is the scale of the scientists who stood at the origins of this direction - M. G. Krein and M. A. Krasnoselskii.

Key words: history of nonlinear functional analysis, Perron-Frobenius theorem, Jentzsch theorem, Krasnosel'skii cone theorem, positive operators, theory of cones, Hammerstein equation, Urysohn equation.

Acknowledgements: The work is supported by RFBR, project 20-011-00402.

For citation: Bogatov E. M. 2020. On the history of the positive operators (1900s - 1960s) and the contribution of M. A. Krasnosel'skii. Applied Mathematics \& Physics, 52(2): 105-127 (in Russian).

DOI 10.18413/2687-0959-2020-52-2-105-127.

1. Введение. $\mathrm{C}$ начала появления первых работ по положительным операторам прошло уже более 100 лет, а середины ХХ в. интерес к этой теме стал расти в геометрической прогрессии (см., например, список литературы в [Amann, 1976]), поэтому её осмысление уже давно назрело. Несмотря на это, историко-математические исследования в данном направлении, по-видимому, не проводились. Кроме того, некоторые западные математики, публикующие монографии по положительным операторам в серьёзных издательствах (см., например, [Aliprantis, Burkinshaw, 2006]), в историческом предисловии не упоминают основополагающие работы о матричных положительных операторах О. Перрона [Perron, 1907б], Ф. Г. Фробениуса [Frobenius, 1908; Frobenius, 1909] и их последователя Р. Ентча с его результатами о положительной разрешимости линейных интегральных уравнений [Jentzsch, 1912].

Трудно также объяснить отсутствие среди «отцов-основателей» обсуждаемой теории таких известных учёных, как М. А. Красносельский, опубликовавший монографию по положительным решениям операторных уравнений ${ }^{2}$ [Красносельский, 1962], В. И. Опойцев [Опойцев, 1977] и многих других отечественных математиков.

Автор постарается в каком-то смысле восстановить историческую справедливость и беспристрастно проследить зарождение и развитие теории положительных операторов, начиная с работ Ш. Ф. Штурма 1830-х гг. и заканчивая исследованиями Красносельского и его учеников до конца 1960-х гг.

Будут найдены ответы на следующие вопросы:

\footnotetext{
${ }^{2}$ Она была переведена на английский язык в 1964 г. издательством Noordhoff.
} 
1. Когда возникла вышеупомянутая теория и чем было обсуловлено её появление?

2. На каком этапе развития теории к исследованиям подключились отечественные математики?

3. Каковы ключевые моменты развития теории положительных операторов?

4. Чем был мотивирован приход М. А. Красносельского в данную область? Каких результатов он достиг, какие инструменты использовал?

5. Как соотносились достижения представителей воронежской математической школы с результатами других учёных, в том числе и иностранных?

6. Какое продолжение получила эта тема в 1960 - 1970-е гг.?

7. Какое воздействие оказала теория положительных операторов на развитие функционального анализа?

Настоящее исследование продолжает цикл работ автора по истории нелинейного функционального анализа (см. [Богатов,2017], [Богатов,2018], [Богатов,2019a], [Bogatov,2020]).

2. Предыстория: положительные матрицы и линейные интегральные уравнения.

В 1907 г. появилась работа немецкого математика Оскара Перрона о положительных собственных значениях матриц с положительными элементами. Основной интерес для нас представляет теорема о существовании наибольшего по модулю, положительного собственного значения матрициь

$A=\left\{a_{i j}>0\right\}$, əde

$$
A x=\lambda x,
$$

которому соотвествует вектор с положительными координатами [Perron, 1907 b; с. 261 ].

Интерес Перрона к положительным матрицам был обусловлен ${ }^{3}$ задачами из алгебры малых колебаний, восходящих к исследованиям французского математика Шарля Франсуа Штурма. Приведём характерный пример [Sturm, 1833; Гантмахер, Крейн, 1950]. Пусть имеется упругая струна, нагруженная массами в некоторых внутренних точках $s_{i}$ (см. рис. 1 ).

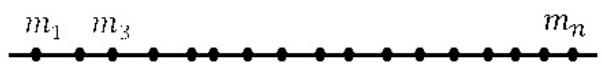

Рис. 1. Упругая струна, нагруженная массами

Fig. 1. Elastic string loaded by masses

Её поперечные перемещения удовлетворяют линейному дифференциальному уравнению

$$
y(x, t)=-\sum_{k=1}^{n} K\left(x, s_{k}\right) m_{k} \ddot{y}_{k},
$$

где $K(x, s)$ - функция влияния струны.

Если предполагать наличие гармонических колебаний, то для отыскания амплитуд прогиба в точках $s_{i}$ мы придём к системе линейных уравнений

$$
u_{i}=p^{2} \sum_{k=1}^{n} a_{i k} m_{k} u_{k},
$$

где $a_{i k}=K\left(s_{i}, s_{k}\right) \geq 0$ есть коэффициенты влияния. Матрица $A=\left\{a_{i k}\right\}$ системы (2) неотрицательна. Результаты Перрона были распространены на матрицы с неотрицательными элементами его соотечественником Фердинандом Георгом Фробениусом [Frobenius, 1908; Frobenius, 1909].

Начало XX в. ознаменовалось интересом к линейным интегральным уравнениям с точки зрения аналогии с матричными уравнениями [Stewart, 2014]. Исследования Фредгольма, Гильберта и Шмидта [Fredholm, 1903; Hilbert, 1904; Schmidt, 1907 a; Schmidt, 1907 b] открывали дорогу для обобщения т. Перрона - Фробениуса на уравнения Фредгольма:

$$
y(s)=\lambda \int_{a}^{b} K(s, t) y(t) d t .
$$

\footnotetext{
${ }^{3}$ См. его статью [Perron, 1907 a], напечатанную в том же номере журнала Mathematische Annalen, что и [Perron, $1907 \mathrm{~b}]$.
} 
Актуальность данного обобщения подтверждалась также тем, что уравнение вида (3) (при непрерывном $K(s, t)>0$ с заменой $d t$ на $d \sigma(t))$ позволяет найти амплитуду колебаний упругого континуума при непрерывном распределении масс [Fredholm,1906; Bateman, 1910, p.420-421]. Указанное исследование было выполнено учеником Фробениуса, немецким математиком Робертом Йентчем в 1912 г. Соответствующая теорема (в несколько упрощённой форме) выглядела так [Jentzsch, 1912; c. 225$]$.

Пусть функция $K(s, t)$ положительна и непрерывна для любых $s, t \in(a, b)$, тогда существует начменьшее по модулю, простое вещественное собственное значение $\lambda_{0}=\min |\lambda|$ уравнения (3), которому соответствует положительная на интервале $(a, b)$ функция $y_{0}(s)$.

3. Нелинейные интегральные уравнения с положительным ядром. Дальнейшее развитие линейной теории уравнений с положительными решениями тормозилось отсутствием общей теории операторов, как таковой (она сложилась к концу 1920-х гг. - см., например, [Bernkopf, 1966; Богатов, Мухин, 2016]). В итоге в течение примерно 15 лет новых результатов о положительной разрешимости уравнения (1) в линейном случае получено не было.

Параллельно с линейной шло развитие теории нелинейных интегральных уравнений [Schmidt, 1908]. Здесь себя проявил представитель московской математической школы Павел Самуилович Урысон. Он обратился к теме положительных решений задач на собственные значения для нелинейных интегральных уравнений в 1918 г. ${ }^{4}$. Не будучи знаком с результатами Ентча, он рассмотрел условия положительной разрешимости уравнения

$$
y=\mu A_{0} y
$$

с нелинейным оператором

$$
A_{0} y(x)=\int_{a}^{b} K(x, s, y(s)) d s .
$$

Проводя свои исследования [Урысон, 1923], Урысон, по-видимому, руководствовался логикой развития самой математики. Однако здесь необходимо отметить, что он был хорошо знаком с задачами гидродинамического характера ${ }^{5}$, приводящими к уравнениям вида $(5)$, что могло оказать своё влияние на выбор объекта исследования.

Используя в качестве основного инструмента метод последовательных приближений, Урысон доказал теорему о существовании положительных решений уравнения (5) в предположении о том, что $K(x, y, 0) \equiv 0$ и что производная $K_{u}^{\prime}(x, y, u)$ непрерывна, положительна и монотонно убывает с возрастанием $u(u \geq 0)$. Он показал, что положительные собственные функции оператора $A_{0}$ существуют при условии, что значения $\mu$ принадлежат некоторому интервалу $\left(\mu_{P}, \mu_{Q}\right)$, где числа $\mu_{P}$ и $\mu_{Q}$ - это наибольшие собственные значения определённых ${ }^{6}$ линейных интегральных операторов $P$ и $Q$. Каждому $\mu \in\left(\mu_{P}, \mu_{Q}\right)$ соответствует единственное положительное решение $y(x, \mu)$ [Урысон, 1951; с. 50-51].

Отметим, что цитированная работа Урысона опередила своё время и не была оценена его современниками по достоинству.

4. Обособление алгебры малых колебаний и выход на теорию моментов. Как уже было отмечено выше, источником исследований Перрона в области матричных уравнений служила теория малых колебаний. Новый круг идей был очерчен одесским математиком Марком Григорьевичем Крейном при более пристальном (про сравнению со Штурмом) изучении свойств матрицы коэффициентов влияния $A_{1}$ струны с точечными массами, закреплённой на обоих концах (см. рис. 1) [Крейн, 1934 b]. Оказалось, что существование осцилляционных свойств связано со следующей особенностью матрицы $A_{1}$ : все миноры любых порядков этой матрицы неотрицательны ${ }^{7}$. В результате исследований, проведённых Крейном совместно с его коллегой, Феликсом Рувимовичем Гантмахером в середине 1930-х гг. [Гантмахер, Крейн, 1935], [Gantmakher, Krein, 1935], [Gantmakher, Krein, 1937], был выделен важный класс так называемых осиилляиионных матрии - вполне неотрицательных квадратных матриц $A$, некоторая степень которых $A^{k}$ является вполне положительной матрицей. При этом был обнаружен следующий факт: основные спектральные свойства этих матриц (вещественность, положительность и простота характеристических чисел, определённые законы чередования знаков у координат собственных векторов и др.) являются общими как для симметричных, так и для несимметричных осцилляционных матриц ${ }^{8}$.

\footnotetext{
${ }^{4}$ Результат был опубликован на 5 лет позже и известности в то время не получил.

${ }^{5} \mathrm{Cм}$. его дневник в книге [Нейман, 1972; с. 71-77].

${ }^{6}$ Подробности будут ниже.

${ }^{7}$ Такие матрицы были названы М. Г. Крейном вполне неотрицателъными.

${ }^{8}$ Тема оказалась настолько актуальной, что монография, подытожившая цикл исследований по этой теме [Гантмахер, Крейн, 1950], была переведена не немецкий и английский языки.
} 
Параллельно с теорией осцилляционных матриц Крейн активно занимался исследованиями по теории моментов [Крейн, 1933; Крейн, 1934 а; Крейн, 1937; Крейн, 1938 а], и этот его интерес был не случаен ${ }^{9}$. Применительно к малым колебаниям нити с бусинами, проблема моментов состоит в определении масс бусин и их расположении таким образом, чтобы частоты колебаний нити имели наперёд заданные значения (подробности см., например, в Дополнениии II книги [Гантмахер, Крейн; 1950]).

Математическая постановка задачи ( $L$-проблема моментов), исследованием которой занимался Крейн, состояла в следующем [Крейн, 1938 а; с. 199]:

Каковы необходимые и достаточные условия, налагаемые на числа $c_{1}, \ldots, c_{n}$, чтобы существовала непрерывная фунциия ограниченной вариащии $\varphi(t)$, удовлетворяющая условиям

$$
\int_{a}^{b} x_{i}(t) d \varphi(t)=c_{i}, \quad i=1, \ldots, n
$$

$$
\Delta \varphi_{1} \leq \Delta \varphi \leq \Delta \varphi_{2} \quad \forall \Delta t
$$

где функции ограниченной вариации $\varphi_{1}(t)$ и $\varphi_{2}(t)$ удовлетворяют условию $\Delta \varphi_{1}<\Delta \varphi_{2} \forall \Delta t$ ?

Проблема моментов волновала умы математиков, в том числе отечественных - П. Л. Чебышева и А. А. Маркова - с конца ХIX в. (см. [Kjeldsen, 1993]; исторические комментарии в [Крейн, Нудельман, 1973]). Поиски путей её решения привели венгерского математика Марселя Рисса к возможности использования идей функционально-аналитического характера [Riesz, 1923]. Он, в частности, показал, что проблема моментов, соответстувющая последовательности $\left\{c_{i}\right\}$ (см. (6)), имеет неубывающее решение $\varphi(t)$ в том и только в том случае, когда линейный функционал $T: f(t) \rightarrow \mathbf{R}$, определённый по правилу

$$
T\left(t^{n}\right)=c_{n}>0
$$

положителен.

Это означало, что любая положительная на отрезке $[a, b]$ линейная комбинация вида

$$
x(t)=\sum_{k=1}^{n} \alpha_{k} t^{k}>0, \quad \alpha_{k} \in \mathbf{R}
$$

преобразуется функционалом $T$ в положительное число: $T(x(t))>0$.

Через 5 лет понятие положительного функционала было обобщено на бесконечномерный случай братом М. Рисса, Фридьешем Риссом (результат был изложен на VIII Международном Математическом конгрессе [Riesz, 1928]). Он назвал функционал $A$, определённый на множестве непрерывных функций $\{f\}$, положительным, если $f>0$ влечёт $A f>0$.

5. Возникновение теории конусов в банаховых пространствах. В конце 1920-х - начале 1930-х гг. функциональный анализ уже выделился в отдельную дисциплину [Bourbaki, 1974; Dieudonne, 1981; Birkhoff, Kreyszig, 1984]. Действуя в рамках функционально-аналитической идеологии и опираясь на подход М. Рисса к решению задач из теории моментов, Крейн ввёл в рассмотрение положительные линейные функционалы, определённые на последовательностях функций $w_{k}(t) \subset E, t \in I$ так, что ${ }^{10}$

$$
w(t)=\sum_{k=1}^{n} \alpha_{k} w_{k}(t)>0 \Rightarrow B w>0 .
$$

Крейн показал [Крейн, 1938а, с. 127], что множество возможных значений функционала $B$ является коническим выпуклым множеством, что помогло свести изучение моментных последовательностей $\left\{c_{n}\right\}$ к исследованию геометрии выпуклых тел в конечномерном пространстве. Более того, содержательная интепретация области определения функционала $B$ позволила Крейну осуществить переход к бесконечномерному случаю: позитивность функиионала в пространстве Е постулировалась, как его способность преобразовывать элементы некоторого конуса $K \subset E$ в неотрицательные (в обычном смысле) элементы [Крейн, 1937; с. 227].

При этом конус в пространстве $E$ представлял собой множество элементов, удовлетворяющее следующим условиям:

\footnotetext{
9Эти же вопросы были предметом научных изысканий Перрона параллельно с изучением свойств положительных матриц [Perron, 1913].

${ }^{10}$ Отметим, что при $\alpha_{k} \geq 0$ линейная комбинация в (7) станет конической комбинацией.
} 
1. $x \in K \Rightarrow \lambda x \in K$ при $\lambda \geq 0$;

2. $x, y \in K \Rightarrow(x+y) \in K$;

3. $x \in K ; x \neq \theta \Rightarrow-x \in E \backslash K$.

Такое определение давало возможность наилучшим образом использовать геометрию банахова пространства $E$, к тому времени уже хорошо разработанную силами львовоской математической школы [Banach, 1932, Гл. VIII; Mazur, 1933]. Здесь, в частности, сыграла свою роль теорема АсколиМазура о существовании опорной к конусу гиперплоскости: она обеспечила наличие хотя бы одного позитивного функционала в $E^{*}$ [Крейн, 1939].

Подтверждением правомерности выделения конусов банахова пространства на роль «эквивалента» множества положительных функций явилась также изоморфность конуса $K$ сепарабельного пространства $E$ множеству функций $\{y(x) \in C[a, b], y(x) \geq 0\}$, доказанная Крейном в 1939 г. [Гросберг, Крейн, 1939]. Крейн также показал, что совокупность положительных функционалов образует конус в сопряжённом пространстве, что, в частности, даёт возможность выйти на доказательство теоремы Маркова о неподвижной точке [Крейн, 1939].

Идея дальнейшего использования конусов банахова пространства была «навеяна» топологическим доказательством теоремы Перрона - Фробениуса, предложенным советским математиком Павлом Сергеевичем Александровым и его немецким коллегой Хейнцем Хопфом [Alexandroff, Hopf, 1935 , с. 480-481]. Они рассмотрели конечномерный оператор $A$, оставляющий октант $K\left(x_{i}>0\right)$ инвариантным, и доказали существование неподвижной точки нелинейного оператора $F$ :

$$
F(x)=\frac{A x}{f(A x)}
$$

на основе теоремы Брауэра ${ }^{11}$ [Brouwer, 1911].

Однако при переходе в бесконечномерное пространство нужна была другая теорема о неподвижной точке ${ }^{12}$. Теорема о том, что вполне непрерывный оператор $A$, преобразующий выпуклое множество банахова пространства $E$ в себя, имеет неподвижную точку, была доказана в 1930 м г. польским математиком Юлиушем Шаудером [Schauder, 1930]. Для применения теоремы Шаудера в контексте доказательства существования решений операторных уравнений вида

$$
A x=\lambda x, \quad x \in K \subset E,
$$

удобнее всего было иметь дело с инвариантными относительно конуса операторами: $A K \subset K$. Первыми это заметили М.Г. Крейн [Крейн, 1939] и его ученик Моисей Аронович Рутман [Рутман, 1938]. Практически сразу свойство $A K \subset K$ было включено в определение положительного оператора [Rutman, 1940, с. 94]. Строго говоря, линейный оператор $A$ был назван положительным, если

1. $A K \subset K, K \subset E$;

2. $\exists x_{0} \in K, c>0 ; c \in \mathbf{R}: A x_{0}-c x_{0} \in K$.

Использование идеи Александрова-Хопфа и аналогов формулы (8) помогли Рутману доказать операторный аналог теорем Перрона-Фробениуса и Ентча для вполне непрерывных линейных операторов, оставляющих инвариантным воспроизводящий ${ }^{13}$ конус $K \subset E$.

Находясь под влиянием теории осцилляционных матриц, Рутман дал определение вполне позитивного оператора, некоторая натуральная степень которого преобразует любой граничный элемент конуса в его внутренний элемент ${ }^{14}$ [Rutman, 1940, с. 65]

$$
\forall(u \in \partial K) \exists(n \in \mathbf{N})\left[A^{n} u \in K\right] .
$$

Такое нововведение позволило усилить теорему Ентча следующим образом:

\footnotetext{
${ }^{11} \mathrm{O}$ существовании неподвижной точки непрерывного отображения, преобразующего выпуклое множество $S \subset R^{n}$ в себя. В данном случае роль $S$ играл симплекс, получающийся при пересечении гиперплоскости $f(x)=1$ с октантом $K$.

${ }^{12}$ Историю метода неподвижной точки см., например, в [Богатов, 2018; Богатов, 2019 а].

${ }^{13}$ Конус $K$ называется воспроизводящим, если любой элемент $z \in E$ представляется в виде разности $z=x-y$, где $x, y \in K$. Это понятие (как и многие другие геометрические понятия теории конусов) принадлежат Крейну. Оно впервые появилось в его неопубликованной довоенной монографии «Теория конусов в пространстве Банаха и её приложения»- см. ссылку в работе [Крейн, Рутман, 1948, с. 7].

14 Здесь предполагается, что конус $K$ имеет внутренние элементы, то есть телесен.
} 
Вполне непрерывный линейный оператор $A$, вполне позитивный относительно конуса $K$, имеет в этом конусе один и толъко один собственный вектор. Соответствующее ему собственное значение является наибольшим по модулю собственным значением оператора $A$.

Поскольку теорема Шаудера не предполагала линейности оператора $A$ в уравнении (9), логично было бы сделать шаги в определении нелинейных положительных операторов и доказательстве разрешимости соответствующих уравнений. Однако естественному ходу событий помешала война и до середины 1950 -х гг. ${ }^{15}$ новые результаты в данной области в СССР не публиковались.

С другой стороны, топологические идеи Александрова и Хопфа получили распространение и на Западе, в результате чего в 1944 г. вышла работа американского математика немецкого происхождения Эриха Роте [Rothe, 1944]. Он рассмотрел спектральную задачу $A x=\lambda x$ в гильбертовом пространстве $H$ и доказал аналог теоремы Ентча для нелинейных вполне непрерывных операторов, заданных на сфере положительных функций пространства $H$, опираясь на соотношение вида (8), теорему Шаудера и геометрию пространства $H$.

Роте, по-видимому, не был знаком с цитированными выше работами Крейна и Рутмана.

6. Становление теории конусов. Нелинейные положительные операторы. Подход, предложенный Роте, не допускал обобщение на произвольные банаховы пространства даже в линейном случае. В то время, как всестороннее исследование геометрии банаховых пространств с конусом, результаты которого были изложены в большой статье ${ }^{16}$ [Крейн, Рутман, 1948], заложили основы для распространения идей теории положительньх операторов в функциональном анализе и его приложениях.

Прежде, чем перейти к нелинейным задачам, отметим, что Крейн и Рутман смогли ослабить требования к линейному оператору $A$ в $(8)$ и (9), заменив полную непрерывность - непрерывностью в слабой топологии для случая, когда пространство $E$ - рефлексивно [Крейн, Рутман, 1948; Теорема 4.1]. Это стало возможным благодаря введению в сопряжённое пространство $E^{*}$ топологии, порождённой системой окрестностей $U_{\varepsilon}\left(f_{0}\right)$ вида

$$
U_{\varepsilon}\left(f_{0}\right)=\left\{f \in E^{*}:\left|f\left(x_{i}\right)-f_{0}\left(x_{i}\right)\right|<\varepsilon ; \quad i=1,2, \ldots, n\right\},
$$

где $f_{0} \in E^{*}$, и применению теоремы Тихонова ${ }^{17}$ о неподвижной точке [Tychonoff, 1935] с учётом того, что всякая замкнутая сфера в $E^{*}$ представляет собой бикомпакт в указанной (слабой) топологии.

Нелинейный операторный аналог теоремы Ентча был доказан Рутманом для положительных операторов, удовлетворяющих условию монотонности ${ }^{18}$

$$
x \succ y \Rightarrow A x \succ A y \quad \text { forall } x, y \in K
$$

и ограничение на рост значений оператора $A$ вдоль некоторого луча внутри конуса:

$$
\exists(u \in K ; \quad c>0, \varepsilon>0)[A(t u) \succ c t u] \forall(t \in[0, \varepsilon]) .
$$

В одномерном случае условие Рутмана (11) допускает геометрическую интерпретацию (см. рис. 2 , $v=t u)$.

Требования (10)-(11) к нелинейному оператору $A$ позволили Рутману сделать заключение о том, что при их выполнении задача (9) всегда имеет положительное решение $(\lambda>0, x \in K)$, причём норма элемента $x$ может выбрана произвольно [Крейн, Рутман, 1948; с. 92].

С конусной тематикой был хорошо знаком и другой ученик М. Г. Крейна, Марк Александрович Красносельский, работавший вместе с Крейном в Институте Математики АН УССР с 1947 по 1952 г. [Боголюбов, Ишлинский, Канторович, 1981]. В то время Красносельский разрабатывал топологические методы теории нелинейных интегральных уравнений, и теория положительных операторов входила в круг его научных интересов [Красносельский, 1951].

Помимо семинаров Крейна по функциональному анализу, он посещал также семенары Н. Н. Боголюбова и Н. М. Крылова по нелинейной механике. Отправными пунктами исследований Красносельского являлись упомянутый выше результат Урысона $^{19}$, а также задача об определении форм потери устойчивости шарнирно-опёртого стержня переменной жёсткости, сводящаяся к уравнению Урысона (4)-(5).

\footnotetext{
${ }^{15}$ В 1947 г. по этой теме вышла совместная работа академика Н. Н. Боголюбова и младшего брата М. Г. Крейна Селиам Крейна [Боголюбов, Крейн, 1947].

16 Эта статья вошла в «золотой фонд» достижений отечественной математики.

${ }^{17}$ Если $X$ - выпуклый бикомпакт, в локально-выпуклом линейном топологическом пространстве, то при непрерывном отображении $X$ в себл существует неподвижная точка.

${ }^{18} \mathrm{~B}$ неравенстве (10) $x \succ y$ равносильно тому, что $x-y \in K$. Тем самым в пространство $E$ вводится упорядочивание.

${ }^{19} \mathrm{~K}$ тому времени был опубликован сборник трудов Урысона [Урысон, 1951], и Красносельский был одним из первых, кто обратил должное внимание на его статью по нелинейным интегральным уравнениям.
} 


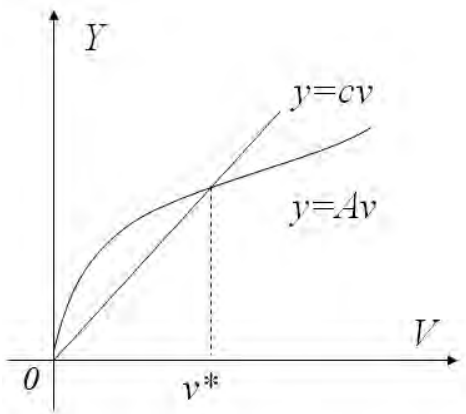

Рис. 2. Геометрический смысл условия Рутмана - существование пересечения вогнутой функции с наклонной прямой

Fig.2. The geometric meaning of the Rutman condition is the intersection of a concave function with an oblique line

7. Вклад М. А. Красносельского в теорию положительных операторов: 1950-е 1960-е гг.

... Создание и развитие теории конусов во многом связано с научной деятельностъю М. А. Красносельского, которому принадлежит серия ярких результатов, составляющих каркас теории, служащих источником её роста виирь и вглубъ ${ }^{20}$.

Красносельский не ограничился применением теории конусов к доказательству теорем существования решений уравнений вида (9). Он стал рассматривать эту теорию, как дополнительную возможность (наряду с вариационными методами, теорией ветвления и теорией степени отображения) для изучения качественных свойств решений уравнения (9) для нелинейных операторов $A$.

Для дальнейшего целесообразно выделить основные линии развития теории конусов в работах Красносельского до конца 1960-х гг.:

1. Введение в расмотрение монотонных минорант.

2. Получение аналогов утверждений теории Урысона.

3. Проверка теории конусов на модельных задачах нелинейной механики.

4. Совмещение методов теории конусов с другими методами нелинейного анализа.

5. Расширение и углубление теории конусов.

6. Выход на приложения.

Перейдём к линии 1. Теорема Рутмана имела ограниченную область применения: положительные операторы, удовлетворяющие её условиям, обязательно должны быть монотонными. Понятно, что в общем случае нелинейность не обязана быть монотонной (например, если ядро имеет вид $K(s, u) \equiv s \cdot \sin (u))$ и здесь нужен какой-то другой инструмент для исследований.

Подсказка для выхода из положения нашлась в статье Урысона [Урысон, 1951, с. 239], который ввёл в рассмотрение функции $P(x, s)$ и $Q(x, s)$ (ядра линейных операторов), удовлетворяющие следующим условиям

$$
P(x, s)>K_{y}(x, s, y)>Q(x, s) .
$$

С помощью этих ядер (а, следовательно, и соответствующих операторов), и проводилась оценка собственных значений оператора (5). Оценки (12) скорее всего и помогли Красносельскому сформулировать понятие монотонной миноранты ${ }^{21} B$ нелинейного оператора $A$ [Красносельский, 1951], как оператора, удовлетворяющего условиям теоремы Рутмана и неравенству

$$
A x \succ B x .
$$

При этом в качестве миноранты $B$ оказалось удобным использовать линейный положительный ${ }^{22}$ оператор. Позже пришло понимание того, что в ряде случаев для $B$ подходит сильная производная по конусу в нуле оператора $A$ [Красносельский, 1962; с. 119].

\footnotetext{
${ }^{20}$ Цит. по предисловию к книге [Опойцев, Хуродзе, 1984].

${ }^{21}$ Понятие операторной миноранты было впервые, по-видимому, дано Ф. Риссом [Riesz, 1928; p.144].

${ }^{22}$ Красносельский упростил понятие положительного оператора, оставив для него только одно условие: $A K \subset K$.
} 
Для дальнейшего рассмотрения Красносельскому оказалось удобным уточнить понятие вполне позитивного оператора, данного Рутманом (см. выше).

Линейный положительный оператор $A$ был назван Красносельским $u_{0}$-ограниченным ${ }^{23}$, если существует такой (ненулевой) элемент конуса $u_{0} \in K$, что некоторая степень оператора $A$ переводит произвольный элемент конуса и внутрь «конусного отрезка» $\left[\alpha u_{0}, \beta u_{0}\right]$ :

$$
\exists\left(u_{0} \in K, u_{0} \neq \theta\right) \forall(u \in K, u \neq \theta) \exists(n \in \mathbb{N} ; \alpha, \beta>0)\left[\beta u_{0} \succ A^{n} u \succ \alpha u_{0}\right] .
$$

Свойства $u_{0}$-ограниченного оператора помогли доказать аналог Урысоновской теоремы о спектре ${ }^{24}$, при условии, что система (12) заменяется на систему

$$
P \varphi \succ A \varphi \succ Q \varphi, \varphi \in K
$$

где $P, Q: u_{0}$ - ограничены и вполне непрерывны [Красносельский, Ладыженский, 1954, с. 329]. В частности, для оператора Урысона в качестве $P$ подошёл оператор

$$
P \varphi(x)=\int_{a}^{b} K_{y}(x, y, 0) \varphi(y) d y,
$$

а в качестве $Q$ - оператор

$$
Q \varphi(x)=\int_{a}^{b} \lim _{u \rightarrow \infty}\left(\frac{K(x, y, u)}{u}\right) \varphi(y) d y .
$$

[Красносельский, Ладыженский, 1959, с. 121].

Переключимся на линию 2. При исследовании неотрицательной разрешимости неоднородного интегрального уравнения

$$
y(x)=\int_{a}^{b} K(x, s, y(s)) d s+f(x)
$$

Урысон наложил некоторые ограничения на ядро $K(x, s, y)$, основными из которых являются предположения о положительности производной $K_{y}(x, s, y)$ и её убывании при возрастании $y$ [Урысон, 1923, с. 238]. По сути, требования Урысона заключались в предположении вогнутости функции $h(y)=K(x, s, y)$.

Развивая эту идею в рамках теории конусов, Красносельский и Ладыженский получили аналогичные результаты для абстрактных операторных уравнений [Красносельский, Ладыженский, 1959]. При этом вместо обычной вогнутости ими была определена и использована так называемая и

Положительный оператор $A$ был назван $u_{0}$-вогнутым, если он монотонен, переводит произвольный элемент конуса и внутрь конусного отрезка $\left[\alpha u_{0}, \beta u_{0}\right]$ и рост его значений вдоль всех лучей, лежащих в конусе, характеризуется специальным образом:

$$
\begin{gathered}
\forall x \in K\left(x \succ \gamma u_{0}, \gamma>0\right) \forall[\alpha, \beta] \subset(0,1) \exists \eta>0: \\
A(t x) \succ(1+\eta) t A x, t \in[a, b] .
\end{gathered}
$$

При таком определении оператор Урысона $A_{0}$ оказался $u_{0}$-вогнутым для $u_{0} \equiv 1$ в конусах неотрицательных функций пространств $C[a, b]$ и $L^{p}[a, b]$.

Поскольку основным методом, используемым Урысоном в упомянутой выше работе [Урысон, 1923], являлся метод последовательных приближений, естественно было осуществить его развитие в рамках теории конусов. Кроме того, этот вопрос имеет важное практическое значение. Соответствующий результат был получен Красносельским вместе с Бахтиным в 1958 г. [Бахтин, Красносельский, 1958]:

Пусть уравнение $A x=x$ с и $u_{0}$-вогнутым оператором $A$ имеет в конусе $K$ ненулевое решение $x^{*}$. Тогда последовательность $x_{n}=A x_{n-1}$ сходится по $u_{0}$-норме $e^{25} \kappa x^{*}$ при любом выборе начального приближения $x_{0} \in K$.

Таким образом, обсуждаемый метод оказался сходящимся не только при любом выборе начального приближения, но и без предположения о непрерывности оператора $A$.

\footnotetext{
${ }^{23}$ Вполне позитивные операторы могут служить примером $u_{0}$-ограниченных операторов, где $u_{0}-$ любой внутренний элемент конуса [Красносельский, Ладыженский, 1954, с. 326].

${ }^{24}$ Позитивный спектр А принадлежит отрезку $\left[\lambda_{Q}, \lambda_{P}\right]$, где $\lambda_{P}, \lambda_{Q}$ - положителъные собственные значения операторов $P$ и $Q$, которым соответствуют положительные собственные векторы.

${ }^{25}$ По определению, $\|x\|_{u_{0}}=\min _{-\alpha u_{0} \succ x \succ \alpha u_{0}} \alpha$. Из сходимости по $u_{0}$-норме вытекает обычная сходимость; обратное неверно [Бахтин, Красносельский, 1958, с. 18-19].
} 
Применение данной теоремы к уравнению Урысона приводит к следующему (неожиданному) результату: в условиях существования положителъного решения у уравнения (4) к этому решению равномерно сходятся приближения

$$
y_{n}(t)=\mu \int_{a}^{b} K\left(t, s, y_{n-1}(s)\right) d s \quad n=1,2, \ldots
$$

для любой неотрищателъной функиии $y_{n}(t)$, не равной нулю тождественно [Бахтин, Красносельский, 1961, с. 319].

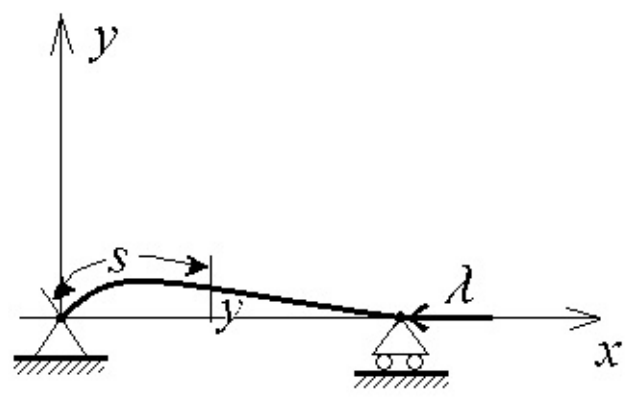

Рис. 3. Продольный изгиб стержня сосредоточенной силой

Fig. 3. Longitudinal bending of the rod by concentrated force

Очередная ветвь развития теории конусов относится к исследованию форм потери устойчивости шарнирно-опёртого стержня переменной жёсткости [Бахтин, Красносельский, 1955]. Оказалось, что нелинейный интегральный оператор, возникающий при описании формы прогиба стержня (см. рис. $3)$ :

$$
\varphi(s)=\lambda \rho(s) \int_{0}^{1} K(s, t) \varphi(t) d t \sqrt{1-\left[\int_{0}^{1} K_{s}^{\prime}(s, t) \varphi(t) d t\right]^{2}},
$$

не обладает свойством $u_{0}$-вогнутости. Это послужило стимулом для отыскания более широкого класса операторов, которые содержали бы класс $u_{0}$-вогнутых операторов и для которых была бы

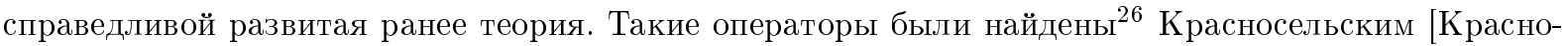
сельский, 1956, с. 288]; они были названы $u_{0^{-}}$монотонными.

Oператор $A$ u-монотонен, если он вогнут $и \varphi_{1} \succ \varphi_{2} \Rightarrow \exists(\alpha>0)\left[A \varphi_{1}-A \varphi_{2} \succ \alpha u_{0}\right]$.

Полученные результаты Красносельскому и Бахтину позволили сделать ряд выводов о том, как меняется форма потери устойчивости стержня при изменении нагрузки на него [Бахтин, Красносельский, 1955, с. 623]:

Теорема. Оператор $A_{1}$ из (17) $u_{0}$-монотонен, $u_{0}=\rho(s)(1-s)$. Критические силь в задаче о продольном изаибе совпадают с собственными значениями линейного оператора

$$
\varphi(s)=\lambda \rho(s) \int_{0}^{1} K(s, t) \varphi(t) d t .
$$

Большей общности удалось достигнуть при введении двух конусов в пространства Банаха: $K$ и $K_{1}\left(K \subset K_{1}\right)$, один из которых - «широкий»- вводился для определения положительности, а второй - «узкий» - для определения монотонности. Это, помимо прочего, позволило Бахтину ввести новый класс вогнутых операторов $\left(\left\{K_{1}, u_{0}\right\}\right.$ - вогнутые операторы), которые содержали $u_{0^{-}}$ вогнутые и $u_{0}$-монотонные операторы, как частный случай [Бахтин, 1957; с. 13], но для которых оставались справедливыми все утверждения теории Урысона.

Перейдём теперь к линии 4. На одной из стадий доказательства сходимости метода последовательных приближений Урысон использовал неравенство [Урысон, 1923; с. 238-239]

$$
\int_{a}^{b} K(x, s, \varphi(s)) d s \leq C K_{y}^{\prime}(x, \xi, 0), \quad \xi \in(a, b) ; \quad C=\text { Const } .
$$

Оно было взято на заметку при развитии М.А. Красносельским теории конусов. Так как в (18) присутствует производная ядра нелинейного оператора $A_{0}$, то ожидаемо использование операторной

\footnotetext{
26 Это произошло, по-видимому, в процессе доказательства обычной монотонности оператора $A_{1}$, стоящего в правой части равенства $(17)$.
} 
производной для обобщения (18) и уточнения оценки оператора $A$ в неравенстве (14). Это уточнение было выполнено с помощью сильной производной Фреше по конусу [Красносельский, Ладыженский, 1954, c. 330]:

$$
P \varphi=A^{\prime}(\theta) \varphi ; \quad Q \varphi=A^{\prime}(\infty) \varphi
$$

где оператор $P$ удовлетворяет условию

$$
\lim _{\|\varphi\| \rightarrow 0} \frac{\|A \varphi-P \varphi\|}{\|\varphi\|}=0,
$$

а оператор $Q-$ условию

$$
\lim _{\|\varphi\| \rightarrow \infty} \frac{\|A \varphi-Q \varphi\|}{\|\varphi\|}=0 .
$$

Условие (19) было названо условием асимптотической линейности оператора $A$.

Например, для оператора Урысона производная Фреше в нуле - это оператор, заданный равенством (15); асимптотическая производная на бесконечности - оператор, заданный равенством (16).

Теперь можно сказать об использовании операторных производных при исследовании спектральных свойств нелинейных операторов. Оказалось, что для $u_{0}$-вогнутых операторов справедлива теорема о топологической структуре спектра [Красносельский, Ладыженский, 1954, с. 331; 338]:

Пусть $A-u_{0}$-вогнутый, вполне непрерывный оператор, асимиптотически близкий $к$ линейно-

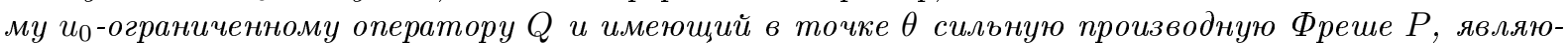

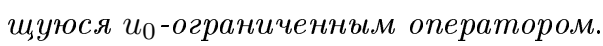

Тогда позитивный спектр оператора А полностъю заполняет интервал $\left(\lambda_{P}, \lambda_{Q}\right)$. Кроме того, положительные собственные векторы оператора А образуют непрерывную ветвъ, выходящую из $\theta$ и уходлщую в бесконечность ${ }^{27}$, причём каждому собственному значению оператора $A$ из позитивного спектра соответствует единственный положителъный собственный вектор $v \neq \theta$.

При рассмотрении вопроса о положительных решениях уравнений с операторами, зависящими om параметра, наиболее естественным является применение топологических методов, в том числе принципа топологического продолжения. Здесь Красносельский использовал ранее разработанную им теорию вращения векторных полей в банаховых пространствах [Красносельский, 1956; Гл. II]. Это позволило, в частности, сравнительно просто доказать разрешимость уравнения

$$
x=A(x, \mu)
$$

и теорему о том, что решения уравнения (20) образуют непрерывную ветвь при условиях существования сильных производных $A^{\prime}(\theta, \mu)$ и $A^{\prime}(\infty, \mu)$ [Красносельский, 1956, Гл. V, §2], а также исследовать бифуркационное значение параметра $\mu$ в (20).

Кроме того, метод Бахтина-Красносельского позволил получить малые ненулевые решения $x(\lambda)$ уравнения

$$
\lambda A x=x
$$

при $\lambda \approx \lambda_{0}\left(\lambda>\lambda_{0}\right)$, как предел последовательных приближений

$$
x_{n}(\lambda)=\mu A x_{n-1}(\lambda) \quad n=1,2, \ldots
$$

[Бахтин, Красносельский, 1961, с. 329], где $\mu$ находится в окрестности $\lambda$.

Перейдём к линии 5. Применяемые Красносельским топологические методы (см. выше) восходят к польскому математику Юлиушу Шаудеру [Schauder, 1930] и голландскому учёному Лёйтзену Брауэру [Brouwer, 1911]. Их идеи были развиты Красносельским в его первой монографии [Красносельский, 1956] и нашли своё продолжение в теории конусов в виде следующей теоремы ${ }^{28}$ [Красносельский, 1960; с. 527].

Пусть вполне непрерывный оператор А сжимает или растягивает конус $K$. Тогда $A$ имеет в конусе $K$ по крайней мере одну ненулевую неподвижную точку.

Терминология, использованная в теореме, требует пояснения.

Оператор $A(A \theta=\theta)$ сжимает конус $K$ (см. рис. 4$)$, если $\exists(R>0, r>0)$ :

$$
x-A x \notin K, \quad x \in K, \quad\|x\| \geqslant R ;
$$

\footnotetext{
${ }^{27}$ Множество положительных собственных векторов оператора $A$ образует по определению непрерывную ветвъ, выходящую из $\theta$ и уходящую в бесконечностъ, если не пусто пересечение этого множества с границей любого ограниченного открытого множества, содержащего $\theta$ [Красносельский, 1951, с. 482].

${ }^{28}$ Специалист по нелинейному анализу, профессор М.К. Квонг (КНР), указывает на то, что данная теорема может быть интерпретирована за рамками метрического восприятия и тем самым поставлена в один ряд с теоремами о неподвижной точке Брауэра и IIаудера [Kwong, 2008; p.6].
} 


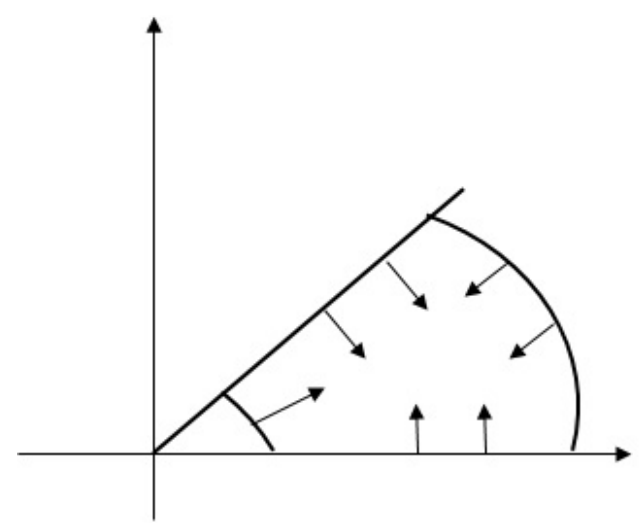

Рис. 4. Сжатие конуса $\mathrm{K}$

Fig. 4. Cone K compression

$$
A x-x \notin K, \quad x \in K, \quad\|x\| \leqslant r .
$$

Оператор $A(A \theta=\theta)$ растлгивает конус $K$ (см. рис. 5), если

$$
\begin{aligned}
& A x-x \notin K, \quad x \in K, \quad\|x\| \geqslant R ; \\
& x-A x \notin K, \quad x \in K, \quad\|x\| \leqslant r .
\end{aligned}
$$

Отметим, что непосредственная проверка условий (22) и (23) представляет собой существенные трудности, в то время как свойства (21) и (24) могут быть выведены, к примеру, из монотонности нормы [Красносельский,1960; с. 527]. Для преодоления этих трудностей Красносельский опять задействовал метод минорант $\left(A_{-}\right)$и мажорант $\left(A_{+}\right)$. Идея заключалась в том, что

$$
\text { если } A x \succ A_{-} x \text {, то }\left[\left(A_{-} x-x\right) \notin K \Rightarrow(A x-x) \notin K\right] \text {. }
$$

Аналогичная импликация справедлива и для мажоранты $A_{+}$. Это позволило переключиться на проверку более простых, по сравнению с (22) и (23), условий для линейного, $u_{0}$-ограниченного оператора $B$ [Красносельский,1960; с. 528].

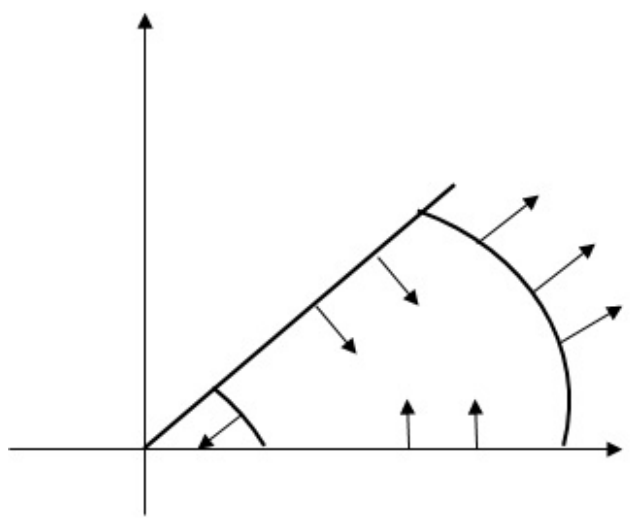

Рис. 5. Растяжение конуса K

Fig. 5. Cone $\mathrm{K}$ extension

Новый принцип неподвижной точки оказалось особенно удобным применять для тех нелинейных уравнений вида

$$
A x=x,
$$

в которых оператор $A$ дифференцируем в нуле и на бесконечности (см. линию 4$)$.

Приведём одну из двух теорем (вторая формулируется аналогично).

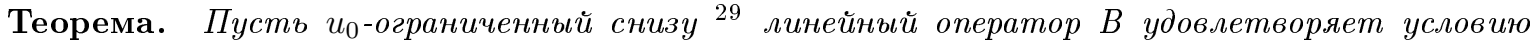
$B u_{0} \succ(1+\varepsilon) u_{0}$, где $\varepsilon>0$. Тогда $B x-x \notin K \forall x \in K, \quad x \neq \theta$.

\footnotetext{
${ }^{29}$ Оператор $B$ был назван Красносельским $u_{0}$-ограниченным снизу, когда каждому $x \in K, x \neq \theta$ соответствуют такие числа $p \in \mathbf{N}, \alpha \in \mathbf{R}$, что $B^{p} x \succ \alpha u_{0}$ [Красносельский, 1960; с. 528].
} 
Подчеркнём, что конусная теорема о неподвижной точке Красносельского допускает обобщения на тот случай, когда уравнение (14) имеет несколько неподвижных точек [Красносельский,1962; с. 164]. В частности, наличие нескольких решений уравнения Гаммерштейна

$$
y(t)=\int_{\Omega} K(t, s) f(s, y(s)) d s
$$

в конусе неотрицательных функций пространства $C(\Omega)$ будет вытекать (в условиях «обобщённой» теоремы о неподвижной точке) из того, что функция $f(s, y)$ содержит участки быстрого и медленного роста по $y$ [Красносельский, Стеценко, 1963; с. 123].

Линия 6 будет представлена в краткой форме.

Перечислим наиболее интересные, с нашей точки зрения, приложения ${ }^{30}$ теории положительных операторов, которые не были упомянуты выше и которые вошли в монографию Красносельского [Красносельский, 1962; Гл. 7]:

1. Доказаны теоремы существования и единственности положительных решений задачи Дирихле для эллиптических уравнений второго порядка в конусе неотрицательных функций и исследованы некоторые свойства этих решений для уравнений, зависящих от параметра.

2. Выявлены условия существования положительных решений у системы обыкновенных дифференциальных уравнений первого порядка. Путь решения задачи заключался в нахождении конусов в фазовом пространстве, которые были бы инвариантны по отношению к сдвигам по траекториям системы ${ }^{31}$. Вывод о наличии периодических решений делался на основе существования неподвижной точки при сдвиге, соответствующем изменению времени на величину периода правых частей системы.

3. Найдены условия положительной разрешимости двухточечной кравевой задачи вида

$$
\begin{array}{r}
\ddot{x}+f(t, x, \dot{x})=0, \\
x(0)=x(1)=0
\end{array}
$$

в конусе неотрицательных функций, в зависимости от свойств функции $f(t, u, v)$.

4. Получены условия существования нетривиальных периодических решений автономных систем дифференциальных уравнений второго порядка с трением и без него.

5. Изучен вопрос о положительной разрешимости задачи Дирихле для уравнения Монжа - Ампера:

$$
\frac{r t-s^{2}}{\left(1+p^{2}+q^{2}\right)^{\alpha}}=f(x, y, z, p, q) ; \quad(x, y, z) \in \Omega \subset \mathbf{R}^{3},
$$

где $p=z_{x}, q=z_{y}, r=z_{x x}, s=z_{x y}, t=z_{y y} ; 0 \leq \alpha \leq 1$.

Основная идея - это обращение оператора, стоящего в левой части (26) и выявление его «полезных» свойств в конусе ограниченных неотрицательных функций.

8. Заключение. В последующие годы (середина 1960 - конец 1970-х) исследования нелинейных уравнений методами теории конусов значительно расширились. Отметим здесь отдельно наиболее весомые работы отечественных математиков:

- определение и использование понятия вращения положительного векторного поля - М. А. Красносельский, П. П. Забрейко и др. [Красносельский, Забрейко, 1975, Гл. 4, §3] (1965-1975 гг.);

- развитие метода последовательных приближений для уравнений с монотонными и нерастягивающими операторами - М. А. Красносельский, В. Я. Стеценко [Красносельский, Стеценко, 1969] (1965-1970 гг.);

- построение теории псеводовогнутых и гетеротонных операторов - В. И. Опойцев [Опойцев, 1975; Опойцев, 1978] (вторая половина 1970-х гг.);

- появление новых разновидностей конусов и операторов в них - И. А. Бахтин [Бахтин, 1975; Бахтин, Бахтина, 1976] (середина 1970-х гг.);

- приложение теории конусов к нелинейным задачам устойчивости - В. И. Опойцев [Опойцев, 1977] (вторая половина 1970-х гг.)

\footnotetext{
${ }^{30}$ Их список далеко не полон (см., например [Красносельский, Рутицкий, 1958, Гл. 4, §20]).

31 Эта тема получила своё дальнейшее развитие в монографии [Красносельский, 1966].
} 
и наиболее известные достижения зарубежных учёных:

- перенос теории конусов в локально-выпуклые пространства - Х. Шефер [Schaefer, 1958]-[Schaefer, 1960];

- доказательство существования трёх решений уравнения $A x=x$ в конусе $Г$. Аман [Amann, 1972];

- обобщение «конусной теоремы о неподвижной точке» Красносельского - Т. Бенджамин [Веnјаmin, 1971] и Р. Нуссбаум [Nussbaum, 1973] (ослабление условий на преобразование границы), а также Р. Леггет и Л. Уильямс [Leggett, Williams, 1979] (использование областей более общего вида).

Подчеркнём, что исследования перечисленных выше западных математиков (кроме немецкого учёного Хельмута Шефера) были выполнены гораздо позже перевода монографии М. А. Красносельского на английский язык [Krasnosel'skii, 1964] и их авторы находились, безусловно, под её влиянием.

Таким образом, можно утверждать, что до конца 1970-х гг. советские математики оставались на лидирующих позициях в теории конусов и положителъных операторов.

Подведём итог, перейдя к ответам на оставшиеся вопросы, сформулированные во введении:

1. Ключевой момент для начала развития теории положительных операторов - исследования Штурма малых колебаний механических систем (начало 1830-х гг.) и появление теоремы Перрона - Фробениуса о положительных собственных значениях матриц с положительными элементами (конец 1900-х гг.).

2. Основная причина для возникновения теории конусов в банаховых пространствах - исследования М. Г. Крейна по теории моментов (середина 1930-х гг.).

3. Отправная точка для начала исследований нелинейных положительных операторов - доказательство теоремы Перрона - Фробениуса топологическими методами, восходящими к Л. Брауэру (П. С. Александров, Х. Хопф - 1935 г.).

4. Обособление теории линейных положителъных операторов произошло после опубликования М. Г. Крейном и М. А. Рутманом системообразующей статьи в журнале УМН (1948 г.).

5. Переломный пункт в развитии теории положительных операторов - её превращение в инструмент качественного исследования операторных уравнений (М. А. Красносельский, 1950-е гг.).

6. Оказалось, что теория линейных положительных операторов является фундаментом для развития нелинейной теории, поскольку

а) в нелинейном анализе используются операторы, представляющие собой композицию линейных и нелинейных операторов;

б) линейные операторы удобно использовать в качестве минорант и мажорант;

в) линейные операторы неизбежно возникают при определении операторных производных.

7. За 25 лет своего развития (1945-1970 гг.) теория конусов обогатила функциональный анализ за счёт появления новых

- функциональных пространств;

- типов операторов и функционалов;

- теорем о неподвижной точке;

- методов исследования спектральных свойств операторов;

- применений производных операторов;

• модификаций и применений метода последовательных приближений.

В заключение хотелось бы отметить, что интерес к исследованиям в обсужаемой области до сих пор не ослабел как в России (см., например, [Кубекова, 2001], [Дорохов, 2009]), так и за рубежом ([Wang, 2003], [Webb, 2010]). По-видимому, эта тенденция будет сохраняться и дальше.

Благодарность. Автор выражает признательность профессору Р. Р. Мухину (СТИ НИТУ МИСИС, г. Старый Оскол) за полезные советы; участникам XXV Годичной научной конференции ИИЕТ РАН им. С. И. Вавилова, ХVI международной конференции «Алгебра, теория чисел и дискретная геометрия: современные проблемы, приложения и проблемы истории» и Воронежской 
зимней математической школе С. Г. Крейна - 2020 за внимание к работе и её обсуждение, а также В. П. Богатовой за перевод первоисточников с немецкого языка и помощь в доступе к оригинальным работам.

\section{Список литературы}

1. Ахиезер Н., Крейн М. 1938. О некоторых вопросах теории моментов. Харьков, гос. науч.-техн. изд-во Украины, 256.

2. Бахтин И. А. 1957. Об одном классе уравнений с положительными операторами. М.: ДАН CCCP, 117(1): 13-16.

3. Бахтин И. А. 1975. Конусы в пространствах Банаха. Ч. 1. Воронеж: Воронеж. гос. пед. ин-т, 183.

4. Бахтин И. А., Бахтина А. А. 1976. Конусы в пространствах Банаха. Ч. 2. Воронеж: Воронеж. гос. пед. ин-т, 135.

5. Бахтин И. А., Красносельский М. А. 1955. К задаче о продольном изгибе стержня переменной жёсткости. М.: ДАН СССР, 105 (4): 621-624.

6. Бахтин И. А., Красносельский М. А. 1958. К теории уравнений с вогнутыми операторами. М.: ДАН СССР, 123 (1): 17-20.

7. Бахтин И. А., Красносельский М. А. 1961. Метод последовательных приближений в теории уравнений с вогнутыми операторами. Новосибирск, Сибирский мат. ж., 2.3 : 313-330.

8. Богатов, Е. М., Мухин Р. Р. 2016. Из истории нелинейных интегральных уравнений. Саратов, Изв. вузов. Прикладная нелинейная динамика. 24 (2): 77-114.

9. Богатов Е. М. 2017. Об истории развития нелинейных интегральных уравнений в СССР. Сильные нелинейности. Белгород, Науч. вед. БелГУ. Сер. Матем., Физ., 6 (46): 93-106.

10. Богатов Е. М. 2018. Об истории метода неподвижной точки и вкладе советских математиков (1920-е - 1950-е гг.). Тула, Чебышевский сборник, 19(2): 30-55.

11. Богатов Е. М. 2019. О развитии качественных методов решения нелинейных уравнений и некоторых последствиях. Саратов, Изв. вузов. Прикладная нелинейная динамика, 27(1): 96114.

12. Богатов Е. М. 2019 а. О развитии теории конусов в работах отечественных математиков. XXV Годичная научная конференция ИИЕТ РАН им. С. И. Вавилова. Саратов: Амирит, 224-227.

13. Богатов Е. М. 2019 b. Об истории теории конусов и полуупорядоченных пространств (в контексте развития нелинейного функционального анализа). Алгебра, теория чисел и дискретная геометрия: современные проблемы, приложения и проблемы истории. Материалы XVI междунар. конф., посвящ. 80-летию со дня рождения проф. Мишеля Деза (13-18 мая 2019). Тула, ТГПУ им. Л.Н. Толстого, 322-325.

14. Богатов Е. М. 2020. О развитии теории положительных операторов и вкладе М. А. Красносельского. Воронежская зимняя математическая школа С. Г. Крейна - 2020: материалы международ. конф. Ред. В.А. Костин. Воронеж: Изд.-полиграф. центр «Научная книга», 8489.

15. Боголюбов Н. Н., Крейн С. Г. 1947. Про позитивні цілком неперервні оператори. Киев, Збірник праць Інституту математики Академіі наук УРСР, 9: 130-139.

16. Боголюбов Н. Н., Ишлинский А. Ю., Канторович Л. В. и др. 1981. Марк Александрович Красносельский (к шестидесятилетию со дня рождения). М.: УМН, 36(2) : 215-220.

17. Гантмахер Ф. Р., Крейн М. Г. 1935. Об одном специальном классе детерминантов в связи с интегральными ядрами Kellog'a. М.: Матем. сб., 42(4) : 501-508.

18. Гантмахер Ф. Р., Крейн М. Г. 1950. Осцилляционные матрицы и ядра и малые колебания механических систем. М-Л.: ГИТТЛ, 360.

19. Гросберг Ю. И., Крейн М. Г. 1939. О разложении линейного функционала на положительные составляющие. М.: ДАН СССР, 15(3) : 721-724. 
20. Дорохов А. Н. 2009. Положительные решения нелинейных уравнений в F-пространствах : Дис. ... кандидата физ.-мат. наук : 01.01.01. Воронеж, ВГУ, 94.

21. Красносельский М. А. 1951. Операторы с монотонной минорантой. М., ДАН СССР, 76 (4) : 481-484.

22. Красносельский М. А. 1954. Некоторые задачи нелинейного анализа. М.: УМН, 9:3(61): 57-114.

23. Красносельский М. А. 1956. Топологические методы в теории нелинейных интегральных уравнений. М.: ГИТЛ, 392.

24. Красносельский М. А. 1960. Неподвижные точки операторов, сжимающих или растягивающих конус. М.: Докл. АН СССР, 135(3): 527-530.

25. Красносельский М. А. 1962. Положительные решения операторных уравнений. М.: ФИЗМАТГИЗ, 394.

26. Красносельский М. А. 1966. Оператор сдвига по траекториям дифференциальных уравнений. М.: Наука, 331.

27. Красносельский М. А., Забрейко П. П. 1975. Геометрические методы нелинейного анализа. М.: Наука, 511.

28. Красносельский М. А., Ладыженский Л. А. 1954. Структура спектра положительных неоднородных операторов. М.: ГИТТЛ, Тр. ММО, 3: 321-346.

29. Красносельский М. А., Ладыженский Л. А. 1959. Об объёме понятия $u_{0}$-вогнутого оператора. Казань, Изв. вузов. Матем., 5: 112-121.

30. Красносельский М. А., Рутицкий Я. Б. 1958. Выпуклые функции и пространства Орлича. М.: ГИФМЛ, 271.

31. Красносельский М. А., Стеценко В. Я. 1963. О некоторых нелинейных задачах, имеющих много решений. Новосибирск, Сиб. мат. ж., 4.1: 120-137.

32. Красносельский М. А., Стеценко В. Я. 1969. К теории уравнений с вогнутыми операторами. Новосибирск, Сиб. мат. ж., 10(3): 565-572.

33. Крейн М. 1934 а. Об одном обобщении исследований акад. Маркова о предельных величинах интегралов. М.: Тр. II Всесоюзного матем. съезда, 2: 152-154.

34. Крейн М. 1934 b. Об узлах гармонических колебаний механических систем некоторого специального типа. М.: Мат. сб. 41(2): 339-348.

35. Крейн М. Г. 1937. Про позитивні адитивні функціонали в лінійних нормованих просторах. Харків, Сообщ. Харьков. Математ. об-ва, XIV: 227-237.

36. Крейн М. Г. 1938. Общие теоремы о позитивных функционалах. В сб. Ахиезер Н., Крейн М. О некоторых вопросах теории моментов. Статья II, Харьков, гос. науч.-техн. изд-во Украины, $121-150$.

37. Крейн М. Г. 1938. О позитивных функционалах в линейных нормированных пространствах. В сб. Ахиезер Н., Крейн М. О некоторых вопросах теории моментов. Статья III, Харьков, гос. науч.-техн. изд-во Украины, 151-170.

38. Крейн М. Г. 1938. L-проблема в абстрактном линейном нормированном пространстве. В сб. Ахиезер Н., Крейн М. О некоторых вопросах теории моментов. Статья IV, Харьков, гос. науч.техн. изд-во Украины, 171-199.

39. Крейн М. Г. 1939. О линейных операторах, оставляющих инвариантным некоторое коническое множество. М.: ДАН СССР, 23(8): 749-752.

40. Крейн М. Г., Рутман М. А. 1948. Линейные операторы, оставляющие инвариантным конус в пространстве Банаха. М.: УМН, 3: 1(23) : 3-95.

41. Крейн М. Г., Нудельман А. А. 1973. Проблема моментов Маркова и экстремальные задачи. М.: Наука, 522. 
42. Кубекова Б.С. 2001. Приближенные решения операторных уравнений с монотонными операторами в пространствах с двумя полуупорядоченностями. Дис. ... кандидата физ.-мат. наук : 01.01.01. Ростов-на-Дону, Рост. гос. ун-т., 118.

43. Нейман Л. С. 1972. Радость открытия (математик Павел Урысон). Научная редакция проф. В. А. Ефремовича. М.: Детская литература, 176.

44. Опойцев В. И. 1975. Гетерогенные и комбинированно-вогнутые операторы. Новосибирск, Сиб. мат. ж., 16(4): 781-792.

45. Опойцев В. И. 1977. Равновесие и устойчивость в моделях коллективного поведения. М.: Наука, 248.

46. Опойцев В. И. 1978. Обобщение теории монотонных и вогнутых операторов . М.: МГУ, Тр. MMO, 36: 237-273.

47. Опойцев В. И., Хуродзе Т. А. 1984. Нелинейные операторы в пространствах с конусом. Тбилиси, Изд-во Тбилисского унив., 270.

48. Рутман М. 1938. Об одном специальном классе вполне непрерывных операторов. М.: ДАН CCCP, 18 (9): 625-627.

49. Урысон П. С. 1923. Об одном типе нелинейных интегральных уравнений. М., Матем. сб., 31 : 236-255.

50. Урысон П. С. 1951. Труды по топологии и другим областям математики. т. 1, М.-Л., ГИТТЛ, 512.

51. Alexandroff P., Hopf H. 1935.Topologie. Berlin, Springer-Verlag, 649.

52. Aliprantis C. D., Burkinshaw O. 2006. Positive operators. Dordrecht, Springer Science \& Business Media, V. 119, 376.

53. Amann H. 1972. On the number of solutions of nonlinear equations in ordered Banach spaces. Journal of functional analysis. 11(3): 346-384.

54. Amann H. 1976. Fixed point equations and nonlinear eigenvalue problems in ordered Banach spaces. Philadelphia, SIAM Review, 18(4) : 620-709.

55. Banach S. 1932. Théorie des operations linéaires. Warszawa, Monografje Matematyczne, I, 231.

56. Bateman H. 1910. Report on the history and present state of the theory of integral equations. London, British Ass. Adv. Sci., 345-424.

57. Benjamin T. B. 1971. A unified theory of conjugate flows. London, Philosophical Transactions of the Royal Society of London. Series A, Math. and Phys. Sci., 269(1201): 587-643.

58. Bernkopf M. 1966. The development of function spaces with particular reference to their origins in integral equation theory. Springer, Arch. Hist. Ex. Sci., 3: 1-96.

59. Birkhoff G., Kreyszig E. 1984. The establishment of functional analysis. Amsterdam, Historia math., 11: 258-321.

60. Bogatov E. M. 2020. On the history of variational methods of non-linear equations investigations and the contribution of Soviet scientists (1920s - 1950s). Warszawa, Antiq. Math., 14 (1): to appear.

61. Bourbaki Nicolas. 1974. É léments d'histoire des mathématiques. Paris, Hermann. 3e édition corrigé et augmenté. Paris, Hermann, 376.

62. Brouwer L. E. J. 1911. Über Abbildung von Mannigfaltigkeiten. Leipzig, Math. Annal.,71: 97-115.

63. Gantmacher F. R., Krein M. G. 1935. Sur les matrices oscillatoires. Paris, CR Acad. Sci. Paris, $201: 577-579$.

64. Gantmakher F., Krein M. 1937. Sur les matrices completement non négatives et oscillatoires. Groningen, Compositio mathematica, 4: 445-476.

65. Dieudonné J. 1981. History of functional analysis. Amsterdam, North-Holland publishing company, 316 . 
66. Hilbert D. 1904. Grundzüge einer allgemeinen Theorie der linearen Integralgleichungen. Vierte Mitteilung. Göttingen, Gött. Nachr.: 49-91.

67. Jentzsch R. 1912. Über Integralgleichungen mit positivem Kern. Berlin, J. Reine Angew. Math., 141: $235-244$.

68. Frobenius G. 1908. Über Matrizen aus positiven Elementen, 1. Berlin, Sitzungsber. Königl. Preuss. Akad. Wiss.: 471-476.

69. Frobenius G. 1909. Über Matrizen aus positiven Elementen, 2. Berlin, Sitzungsber. Königl. Preuss. Akad. Wiss.: 514-518.

70. Fredholm I. 1903. Sur une classe d'équations fonctionnelles. Djursholm , Acta Math., 27: 365-390.

71. Fredholm I. 1906. Solution d'un problème fondamental de la théorie de l'élasticité. Stockholm, Ark. Math. Astronom. Fys., 2 (28) : 3-8.

72. Kjeldsen T. H. 1993. The early history of the moment problem. Amsterdam, Hist. Math., 20 (1) : 19-44.

73. Krasnosel'skii M. A. 1964. Positive solutions of operator equations. Groningen , Noordhoff, 381.

74. Krein M. 1933. Über eine neue Klasse von Hermiteschen Formen und über eine Verallgemeinerung des trigonometrischen Momentenproblems. Moscow, Bulletin de l'Acadé mie des Sciences de l'URSS. Classe des sciences mathématiques et na, $9: 1259-1275$.

75. Kwong M. K. 2008. The topological nature of Krasnoselskii's cone fixed point theorem. London, Nonlinear Analysis: Theory, Methods \& Applications. 69 (3): 1-18.

76. Leggett R. W., Williams L. R. 1979. Multiple positive fixed points of nonlinear operators on ordered Banach spaces. Bloomington, Indiana University Math. J. 28 (4) : 673-688.

77. Lützen J. 1984. Sturm and Liouville's work on ordinary linear differential equations. The emergence of Sturm-Liouville theory. Springer-Verlag GmbH, Arch. Hist. Exact Sci., 29 (4): 309-376.

78. Mazur S. 1933. Über konvexe Mengen in linearen normierten Räumen. Lwów, Studia Math., 4: $70-84$.

79. Nussbaum R. D. 1973. Periodic solutions of some nonlinear, autonomous functional differential equations. II. Amsterdam, Journal of Differential Equations, 14(2): 360-394.

80. Padovani F. 2009. Collected Works of Charles François Sturm. Basel, Birkhäuser, 808.

81. Perron O. 1907. Grundlagen f ür eine Theorie des Jacobischen Kettenbruchalgorithmus. Leipzig, Math. Annalen, 64(2) : 1-76.

82. Perron O. 1907. Zur Theorie der Matrices. Leipzig, Math. Annalen, 64 (2): 248-263.

83. Perron O. 1913. Erweiterung eines Markoffschen Satzes über die Konvergenz gewisser Kettenbrüche. Leipzig, Math. Annalen, 74 : 545-554.

84. Riesz M. 1923. Sur le probleme des moments. Troisieme Note. Stokholm, Ark. Mat. Fys., 16 : 1-52.

85. Riesz F. 1928. Sur la dé composition des opérations fonctionnelles linéaires. Bolonia, Atti del Congresso Internazionale dei Matematici, 1928. V. $3: 143-148$.

86. Rothe E. 1944. On non-negative functional transformations. Baltimore, Johns Hopkins University Press, American J. Math., 66(2): 245-254.

87. Rutman M. A. 1940. Sur les opérateurs totalement continus linéaires laissant invariant un certain cone. Moscow, Mat. Sb. (N.S.), 8(50):77-96.

88. Schaefer H. H. 1958. Halbgeordnete lokalkonvexe Vektorräume I. Leipzig, Math. Ann., 135:115-141.

89. Schaefer H. H. 1959. Halbgeordnete lokalkonvexe Vektorräume II. Leipzig, Math. Ann., 138: 254286.

90. Schaefer H. H. 1960. Halbgeordnete lokalkonvexe Vektorräume III. Leipzig, Math. Ann., 141:113142. 
91. Schauder J. 1930. Der Fixpunktsatz in Funktionalräumen. Kraków, Studia Math. 2: 171-180.

92. Schmidt E. 1907. Zur Theorie der linearen und nichtlinearen Integralgleichungen. I Teil. Entwicklung willkürlichen Funktionen nach System vorgeschriebener. Leipzig, Math. Ann., 63: 433-476.

93. Schmidt E. 1907. Zur Theorie der linearen und nichtlinearen Integralgleichungen. II. Teil. Auflösung der allgemeinen linearen Integralgleichung. Leipzig, Math. Ann., 64:161-174.

94. Schmidt E. 1908. Zur Theorie der linearen und nichtlinearen Integralgleichungen. III Teil. Über die Auflösung der nichtlinearen Integralgleichung und die Verzweigung ihrer Losungen. Leipzig, Math. Ann., 65:370-399.

95. Stewart G. W. 2014. FREDHOLM, HILBERT, SCHMIDT Three Fundamental Papers on Integral Equations. URL: http://www.cs.umd.edu/_stewart/FHS.pdf (дата обращения 03.03.2019).

96. Sturm C. 1833. Analyse d'un mé moire sur les propriétés géné rales des fonctions, qui dépendent d'é quations différentielles linéaires du second ordre. Paris, L'Institut. Journ. Acad. et Soc. 11: 219-223.

97. Tychonoff A. 1935. Ein Fixpunktsatz. Leipzig, Math. Ann., 111: 767-776.

98. Wang H. 2003. On the number of positive solutions of nonlinear systems. Amsterdam, J. Math. Analysis Appl., 281(1): 287-306.

99. Webb J. R. L. 2010. Solutions of nonlinear equations in cones and positive linear operators. London, J. of London Math. Soc., 82(2) : 420-436.

\section{References}

1. Akhiezer N., Krein M. 1938. On some issues of the theory of moments. Kharkov, gos. nauch.-tech. izd-vo of Ukraine, 256 (In Russian).

2. Bahtin I. A. 1957. On a class of equations with positive operators. Moscow, Dokl. Akad. Nauk SSSR, 117(1): 13-16 (In Russian).

3. Bahtin I. A. 1975. Cones in Banach spaces. I. Textbook. Voronezh, Voronezh gos. ped. inst., 183 (In Russian).

4. Bahtin I. A., Bahtina A. A. 1976. Cones in Banach spaces. II. Textbook. Voronezh, Voronezh gos. ped. inst., 135 (In Russian).

5. Bahtin I. A., Krasnosel'skii M. A. 1955. On the problem of longitudinal bending of a rod of variable stiffness. Moscow, Dokl. Akad. Nauk SSSR, 105 (4): 621-624 (In Russian).

6. Bahtin I. A., Krasnosel'skii M. A. 1958. On the theory of equations with concave operators. Moscow, Dokl. Akad. Nauk SSSR, 123 (1): 17-20 (In Russian).

7. Bakhtin I. A., Krasnosel'skii M. A. 1961. The method of successive approximations in the theory of equations with concave operators. Novosibirsk, Sibirsk. Mat. Zh., 2: 313-330.

8. Bogatov E. M., Mukhin R. R. 2016. About the history of nonlinear integral equations. Saratov, Izvestiya VUZ. Applied nonlinear dynamics, 24 (2): 77-114 (In Russian).

9. Bogatov E. M. 2017. On the history of the development of nonlinear integral equations in the USSR. High nonlinearities. Belgorod, Scientific bulletin of BelSU. Ser. Mat., Phys., 6 (46): 93-106 (In Russian).

10. Bogatov E. M. 2018. On the history of the fixed point method and the contribution of the Soviet mathematicians (1920s - 1950s.). Tula, Čebyševskij sbornik, 19(2): 30-55 (In Russian).

11. Bogatov E. M. 2019. On the development of qualitative methods for solving nonlinear equations and some consequences. Saratov, Izvestiya VUZ. Applied nonlinear dynamics, 27(1): 96-114 (In Russian).

12. Bogatov E. M. 2019 a. On the development of cone theory in the works of domestic mathematicians. Annual scientific conference of IHST RAS, Saratov: Amirit, 224-227 (In Russian). 
13. Bogatov E. M. 2019 b. On the history of the theory of cones and semi-ordered spaces (in the context of the development of nonlinear functional analysis). Algebra, number theory and discrete geometry: modern problems, applications and problems of history. Materials XVI Int. conf., Tula, TGPU im. L. N. Tolstogo, 322-325 (In Russian).

14. Bogatov E. M. 2020. On the development of the theory of positive operators and the contribution of M. A. Krasnoselskii. Voronezh Winter Mathematical School S. G. Krein 2020: Materials Int. conf. Voronezh, Nauchnaya kniga, 84-89 (In Russian).

15. Bogoliubov N. N. 2005. Collection of Scientific Papers: in 12 vol. Vol. I. Mathematics, 1925-1990. V. S. Vladimirov, A. D. Sukhanov eds. Moscow, Nauka, 775.

16. Bogolyubov N. N. , Ishlinskii A. Yu. , Kantorovich L. V. and others. 1981. Mark Aleksandrovich Krasnosel'skii (on his sixtieth birthday). Moscow, Russian Math. Surveys, 36(2): 205-212.

17. Gantmakher F., Krein M. 1935. Sur une classe spéciale de déterminants ayant le rapport aux noyaux de Kellog. Moscow, Mat. Sb., 42(4): 501-508.

18. Gantmakher F. R., Krein M. G. 1961. Oscillation matrices and kernels and small vibrations of mechanical systems. 2d ed. corr. and expanded. Oak Ridge, 408.

19. Grosberg Yu. I., Krein M. G. 1939. On the decomposition of a linear functional into positive components. Moscow, Dokl. Akad. Nauk SSSR, 15(3): 721-724.

20. Dorokhov A. N. 2009. Positive solutions of nonlinear equations in F-spaces: PhD Thesis. Voronezh, VSU, 94.

21. Krasnosel'skii M. A. 1951. Operators with monotonic minorant. Moscow, Dokl. Akad. Nauk SSSR, $76(4): 481-484$.

22. Krasnosel'skii M. A. 1954. Some problems of nonlinear analysis. Moscow, Uspekhi Mat. Nauk, 9:3(61): 57-114 (In Russian).

23. Krasnosel'skii M. A. 1964. Topological Methods in the Theory of Nonlinear Integral Equations. Oxford, Pergamon, 395.

24. Krasnosel'skii M. A. 1960. Fixed points of cone-compressing or cone-extending operators. Moscow, Dokl. Akad. Nauk SSSR, 135(3): 527-530 (In Russian).

25. Krasnosel'skii M. A. 1962. Polozhitel'nye resheniya operatornykh uravnenii. Moscow, FIZMATGIZ, 394 (In Russian).

26. Krasnosel'skii M. A. 1968. The Operator of Translation Along the Trajectories of Differential Equations (Translations of Mathematical Monographs, Vol 19). Providence, AMS, 294.

27. Krasnosel'skii M. A., Zabreiko P. P. 1984. Geometrical methods of nonlinear analysis (Vol. 263). Berlin ; New York, Springer-Verlag, 409.

28. Krasnosel'skii M. A., Ladyzhenskii L. A. 1954. The structure of the spectrum of positive nonhomogeneous operators. Moscow, GITTL, Tr. Mosk. Mat. Obs., 3: 321-346.

29. Krasnosel'skii M. A., Ladyzhenskii L. A. 1959. The scope of the concept of a $u_{0}$-concave operator. Kazan, Izv. Vyssh. Uchebn. Zaved. Mat., 5 : 112-121.

30. Krasnosel'skii M. A., Rutitski, Ya. B. 1961. Convex functions and Orlicz spaces. Translated from the first Russian edition. Groningen, Noordhoff, 249.

31. Krasnosel'skii M. A., Steysenko V.Ya. 1963. On some nonlinear problems that have many solutions. Novosibirsk, Sibirsk. Mat. Zh., 4.1: 120-137 (In Russian).

32. Krasnosel'skii M. A., Steysenko V.Ya. 1969. On the theory of equations with concave operators. Novosibirsk, Sibirsk. Mat. Zh., 10(3): 565-572 (In Russian).

33. Krein M. 1934. About one generalization of Acad. Markov research on limit values of integrals. Moscow, Proceedings of the 2nd All-Union Mathem. Congress, 2: 152-154 (In Russian).

34. Krein Mark. 1934. Über die Knoten der harmonischen Schwingungen einiger spezieller mechanischer Systeme. Moscow, Mat. Sb., 41(2): 339-348. 
35. Krein M. G. 1937. On the positive additive functionals in linear normed spaces. Kharkiv, Communications Kharkov Math. Soc., 14: 227-237. (In Ukrainian).

36. Krein M. G. 1938. General theorems on positive functionals. In the Coll. Sci. Works Akhiezer N., Krein M. On some issues of the theory of moments. Paper II, Kharkov, gos. nauch.-tech. izd-vo of Ukraine, 121-150 (In Russian).

37. Krein M. G. 1938. On the positive functionals in normed linear spaces. In the Coll. Sci. Works Akhiezer N., Krein M. On some issues of the theory of moments. Paper III, Kharkov, gos. nauch.tech. izd-vo of Ukraine, 151-170 (In Russian).

38. Krein M. G. 1938. L-problem in an abstract linear normed space. In the Coll. Sci. works Akhiezer N., Krein M. On some issues of the theory of moments. Paper III, Kharkov, gos. nauch.-tech. izd-vo of Ukraine, 171-199 (In Russian).

39. Krein M.G. 1939. On linear operators that leave some conic set invariant. Moscow, Dokl. Akad. Nauk SSSR, 23(8): 749-752 (In Russian).

40. Krein M. G., Rutman M. A. 1948. Linear operators leaving invariant a cone in a Banach space. Moscow, Uspekhi Mat. Nauk, 3:1(23): 3-95.

41. Krein M. G., Nudelman A. A. 1977. The Markov moment problem and extremal problems. (Translations of Mathematical Monographs). Providence, AMS, 417.

42. Kubekova B. S. 2001. Approximate solutions of operator equations with monotone operators in spaces with two semi-orders. : PhD Thesis, Rostov-on-Don, Rostov State Univ., 118.

43. Neiman L. S. 1972. The joy of discovery (mathematician Pavel Urysohn). Prof. V.A. Efremovich scientific editorship. Moscow, Detskaya literatura, 176 (In Russian).

44. Opoitsev V. I. 1975. Heterogeneous and combined concave operators. Novosibirsk, Sibirsk. Mat. Zh., 16(4): 781-792 (In Russian).

45. Opoitsev V. I. 1977. Equilibrium and stability in models of collective behavior. Moscow, Nauka, 248 (In Russian).

46. Opoicev V. I. 1978. Generalization of the theory of monotone and concave operators, Moscow, MSU, Tr. Mosk. Mat. Obs., 36: 237-273.

47. Opoitsev V. I., Hurodze T. A. 1984. Nonlinear operators in spaces with a cone. Tbilisi, Izd-vo Tbilisskogo univ., 270 (In Russian).

48. Rutman M. 1938. On a special class of completely continuous operators. Moscow, Dokl. Akad. Nauk SSSR, 18 (9): 625-627 (In Russian).

49. Urysohn P. 1923. Sur une classe d'équations intégrales non lineaires, Moscow, Mat. Sb., 31(2): 236-255.

50. Urysohn P. S. 1951. Works on topology and other areas of mathematics. Vol. 1. Moscow-Leningrad, GITTL, 512 (In Russian).

51. Alexandroff P., Hopf H. 1935.Topologie. Berlin, Springer-Verlag, 649.

52. Aliprantis C. D., Burkinshaw O. 2006. Positive operators. Dordrecht, Springer Science \& Business Media, V. 119, 376.

53. Amann H. 1972. On the number of solutions of nonlinear equations in ordered Banach spaces. Journal of functional analysis. 11(3): 346-384.

54. Amann H. 1976. Fixed point equations and nonlinear eigenvalue problems in ordered Banach spaces. Philadelphia, SIAM Review, 18(4) : 620-709.

55. Banach S. 1932. Théorie des operations linéaires. Warszawa, Monografje Matematyczne, I, 231.

56. Bateman H. 1910. Report on the history and present state of the theory of integral equations. London, British Ass. Adv. Sci., 345-424.

57. Benjamin T. B. 1971. A unified theory of conjugate flows. London, Philosophical Transactions of the Royal Society of London. Series A, Math. and Phys. Sci., 269(1201): 587-643. 
58. Bernkopf M. 1966. The development of function spaces with particular reference to their origins in integral equation theory. Springer, Arch. Hist. Ex. Sci., 3: 1-96.

59. Birkhoff G., Kreyszig E. 1984. The establishment of functional analysis. Amsterdam, Historia math., 11: $258-321$.

60. Bogatov E. M. 2020. On the history of variational methods of non-linear equations investigations and the contribution of Soviet scientists (1920s - 1950s). Warszawa, Antiq. Math., 14 (1): to appear.

61. Bourbaki Nicolas. 1974. É léments d'histoire des mathématiques. Paris, Hermann. 3e édition corrigé et augmenté. Paris, Hermann, 376.

62. Brouwer L. E. J. 1911. Über Abbildung von Mannigfaltigkeiten. Leipzig, Math. Annal.,71: 97-115.

63. Gantmacher F. R., Krein M. G. 1935. Sur les matrices oscillatoires. Paris, CR Acad. Sci. Paris, 201 : 577-579.

64. Gantmakher F., Krein M. 1937. Sur les matrices completement non négatives et oscillatoires. Groningen, Compositio mathematica, 4: 445-476.

65. Dieudonné J. 1981. History of functional analysis. Amsterdam, North-Holland publishing company, 316.

66. Hilbert D. 1904. Grundzüge einer allgemeinen Theorie der linearen Integralgleichungen. Vierte Mitteilung. Göttingen, Gött. Nachr.: 49-91.

67. Jentzsch R. 1912. Über Integralgleichungen mit positivem Kern. Berlin, J. Reine Angew. Math., 141: 235-244.

68. Frobenius G. 1908. Über Matrizen aus positiven Elementen, 1. Berlin, Sitzungsber. Königl. Preuss. Akad. Wiss.: 471-476.

69. Frobenius G. 1909. Über Matrizen aus positiven Elementen, 2. Berlin, Sitzungsber. Königl. Preuss. Akad. Wiss.: 514-518.

70. Fredholm I. 1903. Sur une classe d'équations fonctionnelles. Djursholm , Acta Math., 27: 365-390.

71. Fredholm I. 1906. Solution d'un problème fondamental de la théorie de l'élasticité. Stockholm, Ark. Math. Astronom. Fys., 2 (28) : 3-8.

72. Kjeldsen T. H. 1993. The early history of the moment problem. Amsterdam, Hist. Math., 20 (1) : 19-44.

73. Krasnosel'skii M. A. 1964. Positive solutions of operator equations. Groningen, Noordhoff, 381.

74. Krein M. 1933. Über eine neue Klasse von Hermiteschen Formen und über eine Verallgemeinerung des trigonometrischen Momentenproblems. Moscow, Bulletin de l'Acadé mie des Sciences de l'URSS. Classe des sciences mathématiques et na, $9: 1259-1275$.

75. Kwong M. K. 2008. The topological nature of Krasnoselskii's cone fixed point theorem. London, Nonlinear Analysis: Theory, Methods \& Applications. 69 (3): 1-18.

76. Leggett R. W., Williams L. R. 1979. Multiple positive fixed points of nonlinear operators on ordered Banach spaces. Bloomington, Indiana University Math. J. 28 (4) : 673-688.

77. Lützen J. 1984. Sturm and Liouville's work on ordinary linear differential equations. The emergence of Sturm-Liouville theory. Springer-Verlag GmbH, Arch. Hist. Exact Sci., 29 (4): 309-376.

78. Mazur S. 1933. Über konvexe Mengen in linearen normierten Räumen. Lwów, Studia Math., 4: 70-84.

79. Nussbaum R. D. 1973. Periodic solutions of some nonlinear, autonomous functional differential equations. II. Amsterdam, Journal of Differential Equations, 14(2): 360-394.

80. Padovani F. 2009. Collected Works of Charles François Sturm. Basel, Birkhäuser , 808.

81. Perron O. 1907. Grundlagen f ür eine Theorie des Jacobischen Kettenbruchalgorithmus. Leipzig, Math. Annalen, 64(2) : 1-76. 
82. Perron O. 1907. Zur Theorie der Matrices. Leipzig, Math. Annalen, 64 (2): 248-263.

83. Perron O. 1913. Erweiterung eines Markoffschen Satzes über die Konvergenz gewisser Kettenbrüche. Leipzig, Math. Annalen, 74 : 545-554.

84. Riesz M. 1923. Sur le probleme des moments. Troisieme Note. Stokholm, Ark. Mat. Fys., 16 : 1-52.

85. Riesz F. 1928. Sur la dé composition des opérations fonctionnelles linéaires. Bolonia, Atti del Congresso Internazionale dei Matematici, 1928. V. 3 : 143-148.

86. Rothe E. 1944. On non-negative functional transformations. Baltimore, Johns Hopkins University Press, American J. Math., 66(2): 245-254.

87. Rutman M. A. 1940. Sur les opérateurs totalement continus linéaires laissant invariant un certain cone. Moscow, Mat. Sb. (N.S.), 8(50): 77-96.

88. Schaefer H. H. 1958. Halbgeordnete lokalkonvexe Vektorräume I. Leipzig, Math. Ann., 135: 115-141.

89. Schaefer H. H. 1959. Halbgeordnete lokalkonvexe Vektorräume II. Leipzig, Math. Ann., 138: 254286.

90. Schaefer H. H. 1960. Halbgeordnete lokalkonvexe Vektorräume III. Leipzig, Math. Ann., 141:113142.

91. Schauder J. 1930. Der Fixpunktsatz in Funktionalräumen. Kraków, Studia Math. 2: 171-180.

92. Schmidt E. 1907. Zur Theorie der linearen und nichtlinearen Integralgleichungen. I Teil. Entwicklung willkürlichen Funktionen nach System vorgeschriebener. Leipzig, Math. Ann., 63: 433-476.

93. Schmidt E. 1907. Zur Theorie der linearen und nichtlinearen Integralgleichungen. II. Teil. Auflösung der allgemeinen linearen Integralgleichung. Leipzig, Math. Ann., 64:161-174.

94. Schmidt E. 1908. Zur Theorie der linearen und nichtlinearen Integralgleichungen. III Teil. Über die Auflösung der nichtlinearen Integralgleichung und die Verzweigung ihrer Losungen. Leipzig, Math. Ann., 65:370-399.

95. Stewart G. W. 2014. FREDHOLM, HILBERT, SCHMIDT Three Fundamental Papers on Integral Equations. URL: http://www.cs.umd.edu/_stewart/FHS.pdf (date of the access 03.03.2019).

96. Sturm C. 1833. Analyse d'un mé moire sur les propriétés générales des fonctions, qui dépendent d'é quations différentielles linéaires du second ordre. Paris, L'Institut. Journ. Acad. et Soc. 11: 219-223.

97. Tychonoff A. 1935. Ein Fixpunktsatz. Leipzig, Math. Ann., 111: 767-776.

98. Wang H. 2003. On the number of positive solutions of nonlinear systems. Amsterdam, J. Math. Analysis Appl., 281(1): 287-306.

99. Webb J. R. L. 2010. Solutions of nonlinear equations in cones and positive linear operators. London, J. of London Math. Soc., 82(2): 420-436.

Получена 20.05.2020

Богатов Егор Михайлович - кандидат физико-математических наук, доцент, доцент кафедры высшей математики и информатики Старооскольского технологического института (филиала) Национального исследовательского технологического университета «МИСиС»

мкр. Макаренко, 42, г. Старый Оскол, 309516, Россия;

Доцент кафедры горного дела филиала Национального исследовательского технологического университета «МИСиС»в г. Губкине Белгородской области

ул. Комсомольская, 16, г. Губкин, 309180, Россия

E-mail: embogatov@inbox.ru 